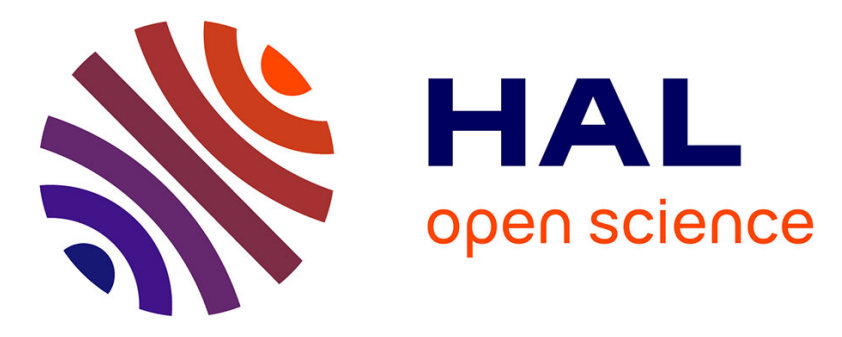

\title{
Convex polygon positioning for homogeneous optical wireless networks
}

Fabián Seguel, Nicolas Krommenacker, Patrick Charpentier, Vincent

Bombardier, Ismael Soto

\section{- To cite this version:}

Fabián Seguel, Nicolas Krommenacker, Patrick Charpentier, Vincent Bombardier, Ismael Soto. Convex polygon positioning for homogeneous optical wireless networks. International Conference on Indoor Positioning and Indoor Navigation, IPIN 2018, Sep 2018, Nantes, France. hal-01890098

\section{HAL Id: hal-01890098 \\ https://hal.science/hal-01890098}

Submitted on 8 Oct 2018

HAL is a multi-disciplinary open access archive for the deposit and dissemination of scientific research documents, whether they are published or not. The documents may come from teaching and research institutions in France or abroad, or from public or private research centers.
L'archive ouverte pluridisciplinaire $\mathbf{H A L}$, est destinée au dépôt et à la diffusion de documents scientifiques de niveau recherche, publiés ou non, émanant des établissements d'enseignement et de recherche français ou étrangers, des laboratoires publics ou privés. 


\section{Convex polygon positioning for homogeneous optical wireless networks}

\author{
Fabian Seguel, Nicolas Krommenacker, \\ Patrick Charpentier and Vincent Bombardier \\ Lorraine University, CRAN, CNRS UMR 7039 \\ Nancy, FRANCE \\ Email: fabian.seguelg@usach.cl
}

\author{
Ismael Soto \\ Electrical Engeneering department \\ Universidad de Santiago de Chile, \\ Santiago, Chile \\ Email: ismael.soto@usach.cl
}

\begin{abstract}
In this paper we present a novel range free method based on connectivity for homogeneous optical wireless networks. Optical attocells deployed homogeneously within an indoor scenario send periodically its position and network architecture information. The mobile node (MN) uses this information in order to self-determine its position by means of solving different convex optimization problems. As a result of this procedure, the feasible localization area is reduced to a convex polygon. A square room of 2.4 meters width and 2.4 meters large with 9 LED lights placed in the ceiling is modeled as the proposed scenario. Our method is compared with two connectivity-based range free algorithms in terms of accuracy. In addition to this, robustness of the method is evaluated using different values on the receiver's Field of View (FOV). Numerical results show that the proposed method outperforms the connectivity based methods in terms of accuracy and robustness.
\end{abstract}

\section{INTRODUCTION}

Indoor localization have gained attention in the recent years due to the rapid growth of micro electromechanical systems and wireless communication networks. The maturity of these technologies deliver a solid base for the implementation of new positioning methods and localization-based services. In addition to this, indoor localization is an attractive research area since its market size is expected to grow from USD 7.11 billion in 2017 to USD 40.99 billion by 2022 [1].

Nowadays, Global Positioning Systems (GPS) is the most used positioning system due to its large coverage and low cost. Nevertheless, GPS based systems are far from being infallible. In metropolitan areas high buildings block the Line-Of-Sight (LOS) signal from the satellites and on indoor scenarios, such as buildings and tunnels complete signal lost is experienced since the signal is not capable to penetrate concrete, metal or ground [2].

Indoor Positioning Systems (IPS) are required to have much more accuracy than outdoor systems making the usage of GPS unfeasible for this type of applications [3]. In order to cover the gap many different systems have been proposed as a complement or a substitute for GPS based positioning. Nowadays, most of the proposed IPS are based on Radio Frequency (RF) signals. Mainly, technologies such as Bluetooth, Wi-Fi, RFID and Zigbee [4]-[6] are used to provide indoor positioning. In particular, WiFi and Bluetooth have been the preferred technologies for domestic implementation since they are commonly integrated in off-the-shelf devices and no dedicated infrastructure is required to implement positioning solutions.

In the recent years, the increment on the usage of wireless communications for diverse types of applications has lead to an increasing demand for bandwidth. Due to the limited unlicensed spectrum provided by RF signals the exploitation of short-wavelength electromagnetic waves for wireless communications has arise as a feasible alternative to satisfy future users demands [7].

Visible Light Communications (VLC) has gained the attention of researchers as an alternative to cope the high bandwidth demand. VLC systems use the visible spectrum ranging from 400 to $800 \mathrm{THz}$ to provide wireless communications and, at the same time, illumination. In addition to the larger bandwidth available in VLC systems, they use the already existing deployed lighting infrastructure with a very few modification making this technology a cost-effective solution [8].

Recently, VLC has been proposed as a possible future solution for $5 \mathrm{G}$ networks. It is expected that in $5 \mathrm{G}$ networks a large number of small radii coverage cells will be used to provide wireless communications at high data rates. Since wireless communication has to be provided ubiquitously, these networks will use spatial diversity in order to solve the problem that the usage small coverage area cells imposes, i.e., many small base stations will be deployed in order to ensure complete coverage [7]. In particular, for VLC networks these small radii coverage cells are often named as optical attocells [9].

As a consequence of the development of VLC based networks, many positioning systems based on VLC have emerged in the recent years. The increasing research on VLC positioning systems has been encouraged by the development of highly accurate VLC-based positioning algorithms verified by experimental set ups. In general, localization algorithms can be classified in two, i.e., range based and range free algorithms. Range based algorithms compute the position of the dumb node (node without knowledge about its position) using one or more characteristics of the received signal such as power, time of arrival, angle of arrival and others [5]. In VLC-IPS many range based methods have been developed and tested in real scenarios [3], [10]. On the other hand, range free 
algorithms use only the connectivity information of the dumb node to compute its position. Range based algorithms are more accurate than range free algorithms. Nevertheless, range based algorithms are less robust than range free algorithms since signal characteristics are easily affected by multipath reflections, shadowing and fading [11].

In literature, VLC-based indoor positioning systems are proposed and implemented on interior building scenario where some assumptions such as square walls, small obstacles and slow dynamic are done. Nevertheless, in scenarios where dynamic big obstacles, non-square shaped surroundings and high fading and scattering exist the performance of the methods will be affected. These type of scenarios can be mainly found in harsh industrial environments such as industrial warehouses where big shelves arranged in narrow corridors can produce shadowing and high multipath component or underground mines tunnels where dust in suspension have strong absorption effects on light waves. Besides, in underground mining scenario big machinery in continuous movement and non-Square shaped walls have to be considered as a source of shadowing and multipath reflection.

In this paper we propose a range free localization algorithm which uses the disconnected neighbor cells information to increase the accuracy and robustness of the localization method.

The work is organized as follows. In Section II the research on VLC-IPS will be analyzed. The methods will be classified in range based and range free. The main characteristics of each approach as well as their advantages and disadvantages will be pointed out. In Section III the system description as well as VLC channel model will be delivered. Definitions of the convex and non-convex constraints used in the proposed method will be formalized. Later on, Section IV will present the proposed method. The numerical results based on computational simulation will be presented and discussed in Section V. Finally, the main conclusions of the study and future research work will be presented in Section VI.

\section{RESEARCH ON ROBUST VISIBLE LIGHT POSITIONING SYSTEMS}

In the recent years different algorithms and test platforms have been developed in order to show the potential of VLC based IPS. Mostly, range based algorithms have been proposed due to their capability to reach high accuracy [3]. The usage of VLC-IPS have been proposed mainly for interior of domiciliary, commercial or public buildings where no big obstacles interrupt or reflect the signal. In addition to this, square rooms with flat walls are considered in most of the cases. In this section the accuracy and robustness of the algorithms that can be found in literature is discussed. We consider the robustness on the accuracy of the algorithm as the resilience on the change of the positioning error against perturbations in the signal propagation such as shadowing, noise and multipath [5].

\section{A. Range based VLC-IPS}

Range based algorithms compute the mobile position based on the characteristics of the received signal. Usually, charac- teristics such as received power, time of arrival and angle of arrival are used to determine the dumb node's position.

Signal characteristics are strongly affected by shadowing, multipath reflections. Since range based methods use signal characteristics to perform the localization, its perturbation will also decrease the algorithm accuracy [12]. Due to this, some studies have been carried out by simulation in order to measure their impact on different proposed methods.

In [13] the impact of multipath on RSS-based IPS was measured by simulation. Positioning accuracy varies from $14.06 \mathrm{~cm}$ when the receiver is considered far from a reflecting surface to $1.52 \mathrm{~m}$ when the receiver is considered to be at a distance of $0.2 \mathrm{~m}$ from walls. In [14] the effect of multipath on positioning was also quantized by simulation when the receiver is positioned in the corner of the room. A positioning error of $9.8 \mathrm{~mm}$ was achieved when no multipath effect was considered whilst the error increases to $1.7 \mathrm{~m}$ when reflections were introduced in the simulation using a reflection factor of of 0.66. The effect of multipath in TOA-based localization was studied in [15]. The Generalized Cramer-Rao lower bound (G-CRLB) was obtained modeling reflections path by gamma distribution. The localization error varies from $0.01 \mathrm{~m}$ to 0.03 $\mathrm{m}$ when LED mean power of $10 \mathrm{~W}$ is considered and different values of $a$ and $b$ are considered in the gamma distributed reflections path.

\section{B. Range free VLC-IPS}

Since range free algorithms use only connectivity information of the dumb node which is less susceptible to be affected by signal disturbances [11] these methods are more robust than range based algorithms. Contrary to the extensive research on VLC based range based localization, range free methods have not been exhaustively studied since they are not capable to provide sufficient accuracy for applications dealing with "lastmeter problem" [3].

Traditionally, in range free methods dumb node uses connectivity information to determine its position. Among different algorithms, convex position estimation, centroid algorithm, Cell ID and APIT are the most commonly used. Nevertheless, to increase the accuracy of these methods a highly dense network is required and, in particular for APIT method a deployment of an heterogeneous network is recommended to improve the granularity of the localization algorithm. Unlike the attention that range based algorithms have had for VLCIPS, range free algorithms have not been study broadly. In [16] a robust approach using VLC-bounding box is proposed. In this paper it is assumed that the mobile node has visibility of at least 4 lights. Only one node position is tested and studies about the error distribution are not carried out. In [17] a cell ID VLC positioning system for underground mines is proposed. Using the proposed method different levels of accuracy can be obtained depending on the coverage area of each cell and the separation between each other. Nevertheless in order to increase the accuracy of the system a high density of the anchor nodes is required. In [18] a experimental Cell ID positioning system using gray code is proposed. The method 
deals with the problem of multiple simultaneous received signals when LED lights use on-off keying (OOK) modulation to transmit its ID and any proposal to increase the accuracy of one hop methods is described in this paper.

\section{SYSTEM DESCRIPTION}

In general, VLC networks are considered as homogeneous networks since LED lights are commonly deployed in a regularly-spaced manner due to illumination constraints as shown in Fig. 1. In the proposed scenario we assume, without losing generality on the method, that each LED light is separated by the same distance in large and width direction of the room, i.e., $D_{x}=D_{y}=D$. In addition to this, LED lights will be deployed in a $3 \times 3$ arrangement as shown in Fig. 1.

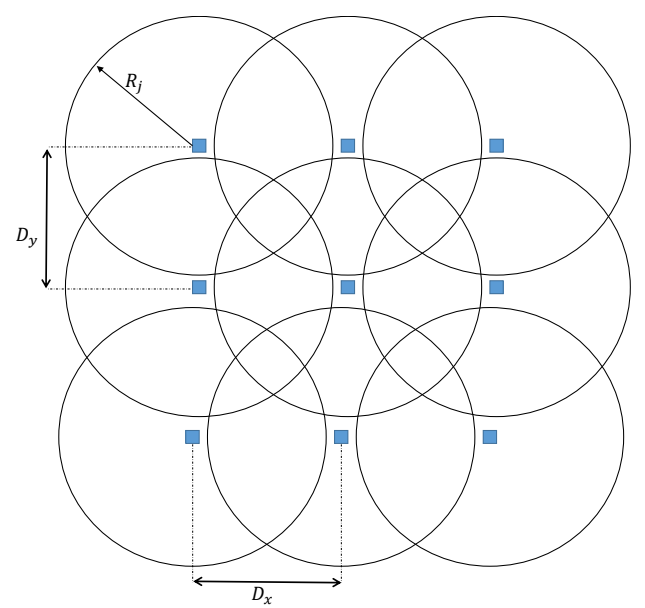

Fig. 1: Scheme of proposed scenario

The received signal power by the Mobile Node (MN) can be modeled as

$$
P_{i}=R_{P D} P_{j} h_{j i}
$$

$P_{i}$ is the received signal power by the $\mathrm{MN} \mathrm{i}$, in our case we consider a single $\mathrm{MN}$, i.e, $i=1$. $R_{P D}, P_{j}$ and $h_{j i}$ are the responsivity of the photodetector, transmitted optical power by LED $\mathrm{j}$ and channel gain of the LED $\mathrm{j}$ to the $\mathrm{MN} \mathrm{i}$ respectively from LED $\mathrm{j}$ to $\mathrm{MN} \mathrm{i}$. When line of sight (LOS) link is considered channel gain can be mathematically expressed as [19]

$h_{j i}= \begin{cases}\frac{\left(m_{l}+1\right) A}{2 \pi d_{j i}^{2}} \cos ^{m_{l}}\left(\varphi_{j i}\right) G\left(\psi_{j i}\right) \cos \left(\psi_{j i}\right) & 0 \leq \psi_{j i} \leq \Psi_{l} \\ 0 & \text { elsewhere }\end{cases}$

in equation (2) $m_{l}$ is the Lambertian order transmission of the LED light, $A$ is referred to the effective area of the $\mathrm{PD}$, the distance between the transmitter $\mathrm{j}$ and receiver $\mathrm{i}$ is denoted as $d_{j i}$. Finally, $\varphi_{j i}$ and $\psi_{j i}$ are the angle of irradiance and the angle of incidence of the signal. The term $G\left(\psi_{j i}\right)=$ $T_{s}\left(\psi_{j i}\right) g\left(\psi_{j i}\right)$ which is the combined gain of the optical filter and the optical concentrator respectively. The gain of the VLC channel is greater than zero if and only if the incidence angle of the signal $\psi_{j i}$ is less or equal to the field of view (FOV) of the optical receiver denoted by $\Psi_{l}$.

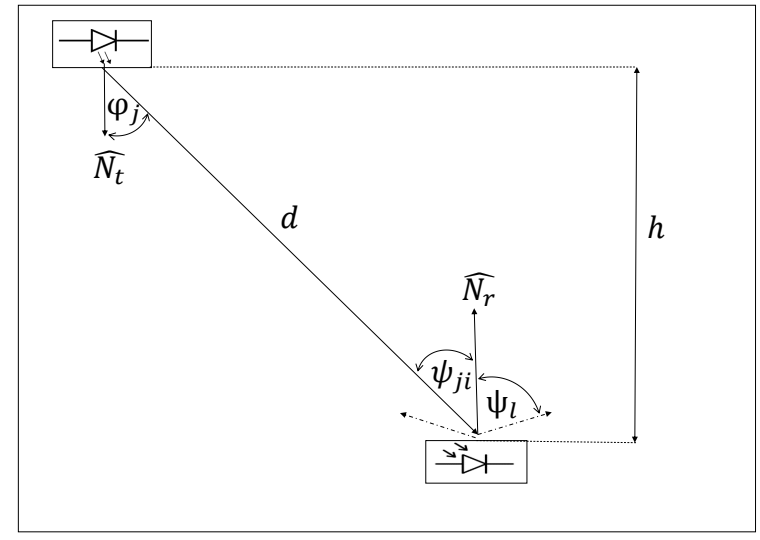

Fig. 2: VLC channel model

From equation (2) can be inferred that the channel gain and, in particular, the coverage radius depends on the incidence angle $\psi_{j i}$ and the FOV of the photodetector. The incidence angle depends on the the relative position between transmitter and receiver. In particular from Fig. 2 it can be seen that different vertical distance between transmitter and receiver $h$ will produce a different coverage radius. Since the FOV of the photodetector is a constant parameter in the system, the impact of their change in the accuracy of the methods will be tested by simulation.

Commonly, lighting devices used for illumination purposes have similar characteristics. Due to this, LED lamps with the same characteristics will be considered for simulation. Parameters of each LED light and their coverage radius are the same, i.e., $R_{0}=R_{1}=\ldots, R_{K}=R$. Two basic definitions will be useful for the next section of the paper. In this work we define the coverage area and the non-covered area of an LED light as two sets in $\mathbb{R}^{2}$

Definition 1: Coverage area: The coverage area of the $j^{\text {th }}$ LED light, $\mathbb{A}_{j}=\left\{\mathbf{x} \in \mathbb{R}^{2} \mid\left\|\mathbf{c}-\mathbf{C}_{j}\right\|_{2}^{2} \leq R_{j}^{2}\right\}$ where $\mathbf{C}_{j} \in \mathbb{R}^{2}$ and $R_{j} \in \mathbb{R}_{+}$are the position and the coverage radius of the LED light respectively.

Definition 2: Non-covered area: The non-covered area of the $j^{\text {th }} \mathrm{LED}, \overline{\mathbb{A}}_{j}=\left\{\mathbf{x} \in \mathbb{R}^{2} \mid\left\|\mathbf{x}-\mathbf{C}_{j}\right\|_{2}^{2}>R_{j}^{2}\right\}$ where $\mathbf{C}_{j} \in \mathbb{R}^{2}$ is the position of the LED light and $R_{j} \in \mathbb{R}_{+}$is its coverage radius.

\section{Convex POLYGon Position (CPP)}

In this section the proposed method based on network architecture is presented. As a practical example of our method we consider that the dumb node is connected to a single LED light with ID 0 as shown in Fig. 4. Information of neighbors cells is received in the beacon transmitted from the LED 0 as 
shown in Fig. 3. The VLC beacon transmitted by each LED light is composed by the ID and position of the received cell as well as their closest neighbors.

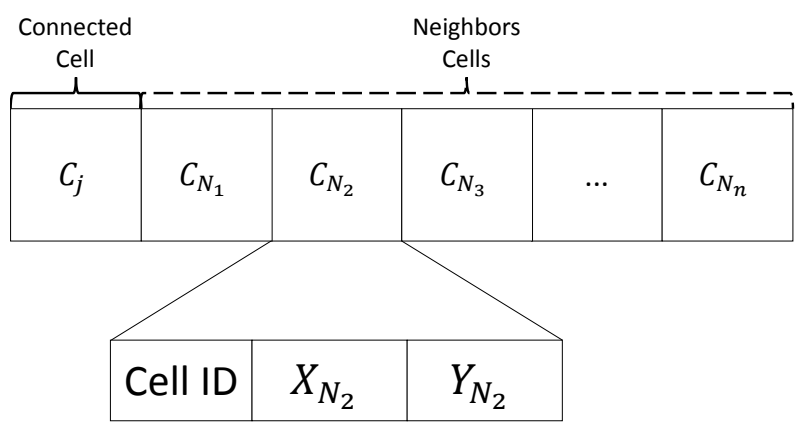

Fig. 3: Beacon transmitted by LED j

Using traditional CPE algorithm the feasible zone in which the MN could be located is represented by the minimum bounding rectangle of the feasible connection-based localization set. The minimum rectangle is found by solving iteratively a linear optimization problem with connectivity constraints. The result of solving the optimization problem is named as bounding box (BB). The bounding box is a convex set in $\mathbb{R}^{2}$, since the rectangle is the intersection of four halfspaces, hence is a convex set in $\mathbb{R}^{2}$. The CPE optimization problem is state as follows

$$
\begin{array}{cl}
\underset{\mathbf{x} \in \mathbb{R}^{2}}{\operatorname{minimize}} & \mathbf{D}^{T} \mathbf{x} \\
\text { subject to } & \left\|\mathbf{x}-\mathbf{C}_{j}\right\| \leq R_{j}
\end{array}
$$

where $\mathbf{D} \in \mathbb{R}^{2}$ is the vector which takes the values $\mathbf{D}=$ $\left[\begin{array}{ll}1 & 0\end{array}\right], \mathbf{D}=\left[\begin{array}{ll}-1 & 0\end{array}\right], \mathbf{D}=\left[\begin{array}{ll}0 & 1\end{array}\right]$ and $\mathbf{D}=\left[\begin{array}{ll}0 & -1\end{array}\right]$ in order to find the minimum and maximum of the bounding box in both axes. The restriction of the problem states that the feasible point $\mathbf{x}$ must lie in the connectivity area.

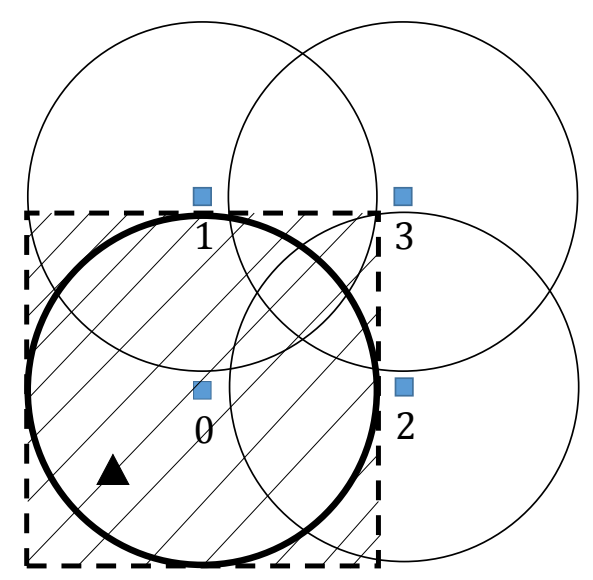

Fig. 4: Convex bounding box for the mobile node position

For the second step, neighbor cells overlapped with the bounding box are found. Since the coverage radius and the central position of each neighbor cell is known by the dumb node overlapping condition can be tested. The overlapped condition state next is tested for each known neighbor and the bounding box set found in the first step.

Definition 3: Overlapping condition: A bounding box set $\mathbb{B B}$ and an optical cell coverage area $\mathbb{A}_{j}$ are considered to be overlapped if the inferior value of the distance between a point $\mathbf{x}$ in the bounding box set, $\mathbf{x} \in \mathbb{B B}$ and the central point of the coverage area set of the $m^{t h}$ LED $\mathbf{C}_{m}$ is inferior to the coverage radius of the $m^{t h}$ cell $R_{m}$. Mathematically this can be expressed as

$$
\mathbb{B B} \cap \mathbb{A}_{m} \neq \phi \Leftrightarrow \inf _{\mathbf{x} \in \mathbb{B B}}\left\|\mathbf{x}-\mathbf{C}_{m}\right\|_{2} \leq R_{m}
$$

Where $\phi$ is the empty set and $\|\cdot\|_{2}$ is the euclidean norm in $\mathbb{R}^{2}$.

The before mentioned overlapping condition can be expressed as convex optimization problem since $\inf _{\mathbf{x} \in \mathbb{B B}}\left\|\mathbf{x}-\mathbf{C}_{m}\right\|_{2}$ is the minimization of the distance between a convex set and a fixed point in $\mathbb{R}^{2}$, i.e., the minimization of the distance between the bounding box set and the center of the coverage area set of the $m^{\text {th }}$ which is a neighbor cell defined as

$$
\begin{array}{cl}
\underset{\mathbf{x} \in \mathbb{R}^{2}}{\operatorname{minimize}} & \left\|\mathbf{x}-\mathbf{C}_{m}\right\|_{2}-R_{m} \\
\text { subject to } & \mathbf{x} \preceq \mathbf{x}_{\max }, \\
& -\mathbf{x} \preceq-\mathbf{x}_{\min }
\end{array}
$$

Where the constraints $\mathbf{x}_{\min }$ and $\mathbf{x}_{\max }$ are the minimum and maximum values of the bounding box set and $R_{m}$ is the coverage radius of the cell. When the fitness function of (5) is strictly negative it means that the cell $m$ is and overlapped cell of the bounding box. Otherwise the cell is not an overlapping cell. We use an overlapping binary function indicator defined as

$$
\text { Overlapping }= \begin{cases}1 & \text { if }\left\|\mathbf{x}^{*}-\mathbf{C}_{m}\right\|_{2}-R_{m}<0 \\ 0 & \text { if }\left\|\mathbf{x}^{*}-\mathbf{C}_{m}\right\|_{2}-R_{m} \geq 0\end{cases}
$$

where $\mathbf{x}^{*}$ is the optimum of the convex optimization problem states in (5). Since the overlapping condition is tested for every neighbor the feasible area can be reduced subtracting a convex approximation of the intersected sets. As shown in Fig. 5 we consider a linear approximation of the set since the the feasible set lies in the intersection of a finite number of halfspaces. Notice that Cell 3 does not contain useful information even if it is considered as an overlapped cell. Every point of the set $\mathbb{A}_{3}$ is an unfeasible point since it is completely covered by , i.e., $\mathbb{B B B} \cap \mathbb{A}_{3} \subseteq \mathbb{A}_{2} \cup \mathbb{A}_{1}$. Since the $\mathrm{MN}$ is not connected to cell 1 or 2 we can remove cell 3 from the localization process. Then, non connected cells 1 and 2 are used to determine the convex hull of the intersection of the feasible localization sets. The $\mathrm{MN}$ lies in the intersection of three sets, this is $M N \in\left\{\mathbb{B B} \cap \overline{\mathbf{A}}_{1} \cap \overline{\mathbf{A}}_{2}\right\}$. 


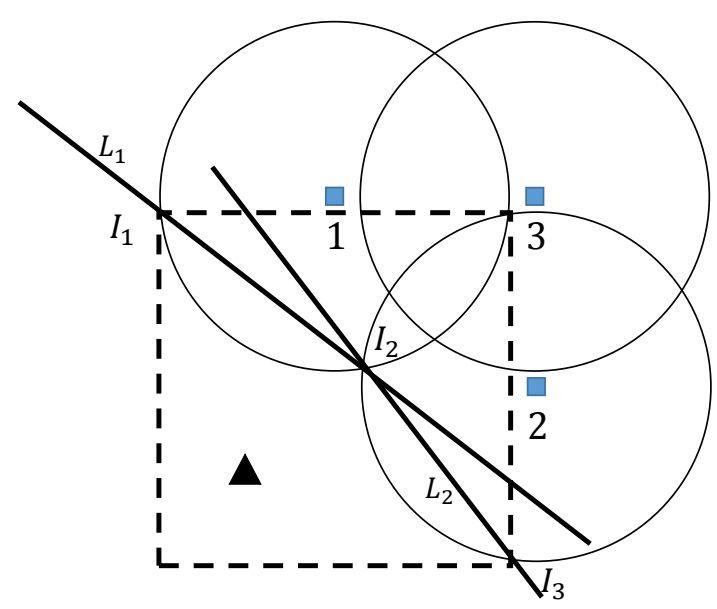

Fig. 5: Considered overlapped optical cells for feasible area reduction and supporting hyperplanes for convex hull

As shown in Fig. 5, $L_{1}$ and $L_{2}$ are the supporting hyperplanes for the convex hull. Note that, $\mathbf{I}_{1}, \mathbf{I}_{2}$ and $\mathbf{I}_{3}$ can be easily found as the intersection points of the sets borders. In detail, $\mathbf{I}_{1}$ is the feasible intersecting point between $\mathbb{A}_{1}$ and the bounding box $\mathbb{B B}, \mathbf{I}_{2}$ is the feasible intersection the two non-coverage are borders $\overline{\mathbb{A}}_{1}$ and $\overline{\mathbb{A}}_{2}$ and finally $\mathbf{I}_{3}$ is the feasible intersection point between the bounding box and the non coverage border of $\overline{\mathbb{A}}_{2}$. Due to this, the problem can be formulated as finding the feasible intersection points between the considered sets borders.

Once the intersecting points are found the rectangular bounding box can be replaced by a convex polyhedron as shown in Fig. 6. The feasible localization polyhedron will have as many vertices as feasible intersecting points between the set borders.
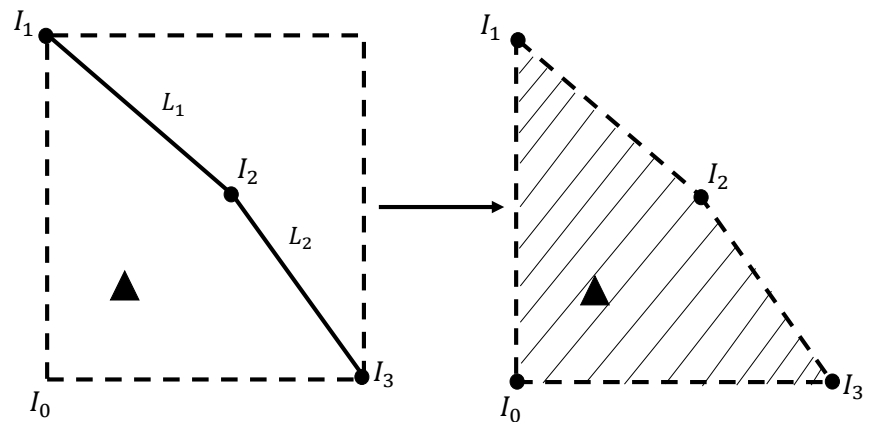

Fig. 6: Reduction of feasible localization area from bounding box rectangle to non-connectivity based polyhedron

Finally, in order to determine the estimated position of the mobile node point free localization will be used.

This approach minimize the euclidean distance between the mobile node and the vertices of the polyhedron, i.e.,

$$
\underset{\mathbf{x} \in \mathbb{R}^{2}}{\operatorname{minimize}} \sum_{j=0}^{K-1}\left\|\mathbf{x}-\mathbf{I}_{j}\right\|_{2}^{2}
$$

The solution in this case is any median of the fixed points

$$
\left(\hat{\mathbf{x}}_{M N}\right)=\left(\frac{1}{K} \sum_{j=0}^{K-1} \mathrm{I}_{x_{j}}, \frac{1}{K} \sum_{j=0}^{K-1} \mathrm{I}_{y_{j}}\right)
$$

Where $K$ is the total number of feasible vertices of the polyhedron, $\mathrm{I}_{x_{j}}$ and $\mathrm{I}_{y_{j}}$ are the vector components of the $j^{\text {th }}$ polyhedron vertex. Note that the final position estimation method uses non-connectivity information since $\mathbf{I}_{1}, \mathbf{I}_{2}$ and $\mathbf{I}_{3}$ where found by using non-connected cells information provided by the received beacon.

To solve problem convex optimization problem of (3) and (5) we used CVX, a package for specifying and solving convex programs in MATLAB [20], [21].

\section{NUMERICAL RESULTS}

LEDs distribution shown in Fig. 1 is used for simulation of the proposed method. System parameters are delivered in Table I. The performance of the CPP algorithm using different values of FOV in the receiver side was tested. In addition to this, the proposed method is compared with two traditional range-free algorithms, these are, bounding box and centroid algorithm. In order to obtain numerical results of positioning error a square grid with separation of $0.1 \mathrm{~m}$ is considered in the receiver plane.

TABLE I: System parameters

\begin{tabular}{|l|l|l|l|}
\hline Parameter & Value & Parameter & Value \\
\hline $\begin{array}{l}\text { Semi-angle } \\
\text { half power }\end{array}$ & $70^{\circ}$ & $\begin{array}{l}\text { Lambertian } \\
\text { Order }\end{array}$ & 0.6461 \\
\hline $\begin{array}{l}\text { LED optical } \\
\text { power }\end{array}$ & $10 \mathrm{~W}$ & Area of PD & $10^{-4} \mathrm{~m}^{2}$ \\
\hline $\begin{array}{l}\text { Gain optical } \\
\text { filter }\end{array}$ & 1 & $\begin{array}{l}\text { half angle } \\
\text { FOV }\end{array}$ & $\begin{array}{l}45^{\circ}, 50^{\circ}, 55^{\circ}, \\
60^{\circ} \text { and } 65^{\circ}\end{array}$ \\
\hline $\begin{array}{l}\text { Room dimen- } \\
\text { sions }\end{array}$ & $2.4 \times 2.4 \mathrm{~m}$ & $\begin{array}{l}\text { Distance } \\
\text { between LEDs }\end{array}$ & $0.75 \mathrm{~m}$ \\
\hline $\begin{array}{l}\text { Transmitter to } \\
\text { receiver plane } \\
\text { height }\end{array}$ & $0.7 \mathrm{~m}$ & $\begin{array}{l}\text { Simulation } \\
\text { grid separation }\end{array}$ & $0.1 \mathrm{~m}$ \\
\hline
\end{tabular}

Using the above shown parameters the mean error and the standard deviation were obtained for the CPP algorithm using different FOV angles values. In Fig.7 the response of the proposed method to changes in the FOV parameter is shown.

The results show that for different FOV angles on the receiver side the CPP method obtains similar performance in terms of mean error and standard deviation. In particular, for FOV angles from 90 to 120 degree the proposed method shows similar performance in terms of mean error and standard deviation where the localization method achieves an error of less than $0.4 \mathrm{~m}$. A mean error of less than $0.3 \mathrm{~m}$ is obtained in all proposed scenarios. The maximum standard deviation of the system corresponds to $\mathrm{FOV}=130^{\circ}$ with a value of 0.1459 . 


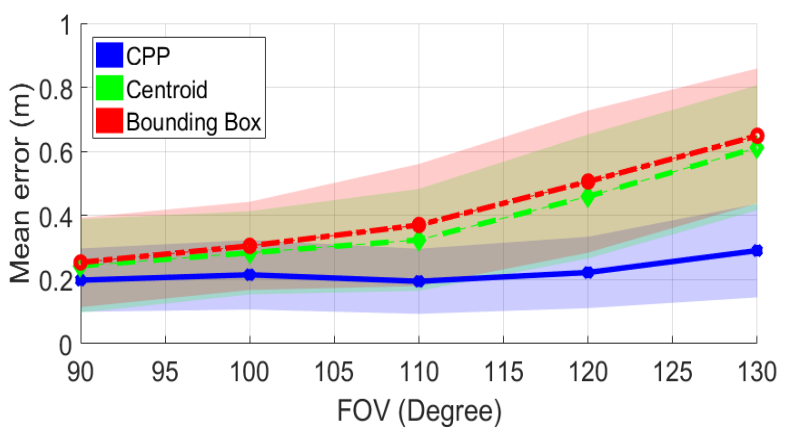

Fig. 7: Error performance and confidence interval of $\mathrm{CP}$, Centroid and CPE for different FOV angles in the receiver side

In addition to this, it can be seen that the accuracy of centroid and CPE methods rapidly decreases as the FOV angle on the receiver increases. for a FOV grater than $130^{\circ}$ the mean error of both methods is grater than $0.6 \mathrm{~m}$ whilst the mean error of the proposed CPP remains lower than $0.4 \mathrm{~m}$. Moreover, using the parameters of Table I, cumulative distribution function (CDF) of the localization error was obtained for the proposed method. In Fig.8 the proposed method is compared with two traditional algorithms, i.e., bounding box and centroid algorithm. The comparison is done for different receiver FOV.

In Fig. 8a the proposed method is compared with bounding box and centroid algorithm for $\mathrm{FOV}=90^{\circ}$. It can be seen that the algorithm achieves an accuracy of $0.4 \mathrm{~m}$ with a $96.48 \%$ of probability whilst centroid and bounding box achieve the same cumulative error with a probability of $82.25 \%$. The proposed methods remains with similar performance for FOV angles from 90 to 110 degrees as shown in fig $8 \mathrm{a}$ and $8 \mathrm{~b}$. The accuracy decreases when a FOV of $130^{\circ}$ is considered, Nevertheless, as it can be seen the proposed method increases its gain over the traditional methods as shown when the the FOV in the receiver side increases making this method more robust to changes in the FOV of the receiver.

\section{CONCLUSIONS}

In this paper a novel range free method for indoor positioning system based on homogeneous VLC network was presented. The proposed method uses the knowledge of lighting deployment to improve the accuracy of the localization. Since the method uses information of non-connected nodes, no extra infrastructure is required to increase the granularity of the range free method, i.e., the system is capable to increase the accuracy without increasing the number of access points deployed.

The proposed method accuracy has shown to be robust to changes in the FOV of the receiver and, under the same conditions, the proposed method outperforms two traditional connectivity based algorithms such as centroid algorithm and CPE.

For future work a real platform will be implemented in order to test the procedure in real scenarios. In addition to this, (a)

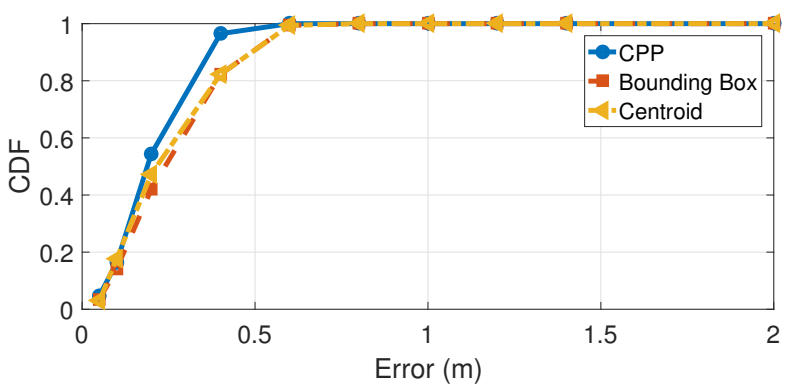

(b)

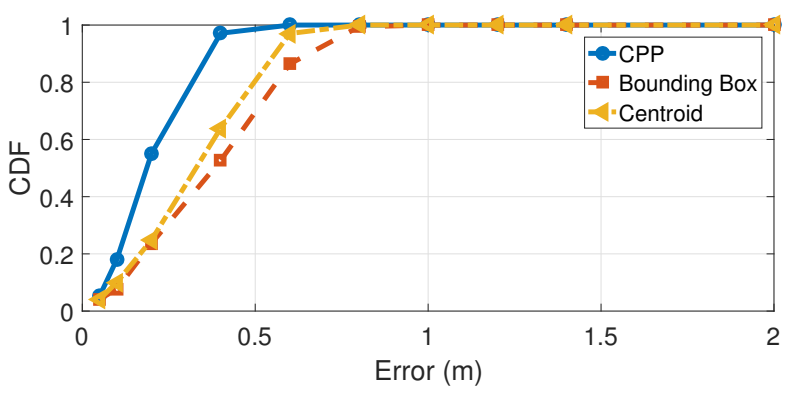

(c)

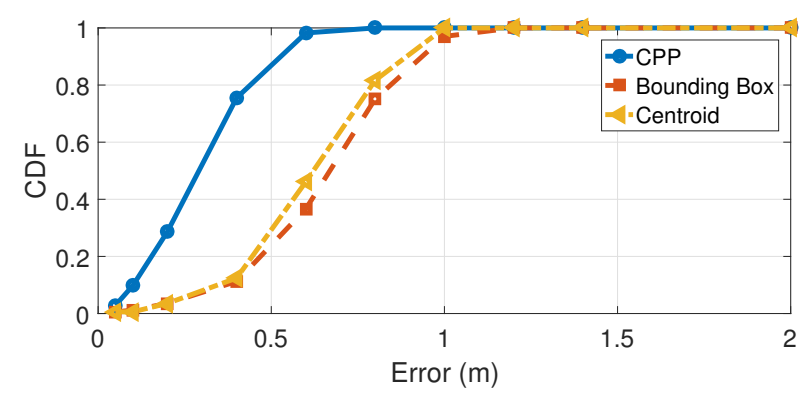

Fig. 8: Cumulative distribution function (CDF) for the proposed method, bounding box and centroid algorithm for (a) $\mathrm{FOV}=90^{\circ}$, (b) $\mathrm{FOV}=110^{\circ}$ and (c) $\mathrm{FOV}=130^{\circ}$

different LED placement configuration will be tested in order to find the one that maximizes the accuracy of the method. Furthermore, the robustness of the method to changes in the transmitter to receiver plane height and LED failure will be analyzed.

\section{ACKNOWLEDGMENT}

The authors acknowledge the financial support of Beca Doctorado Nacional 2016 CONICYT (PFCHA) 21161397 and Fondef PROYECTO IT17M10012.

\section{REFERENCES}

[1] BusinessWire, "Retail Indoor Location Market Breaks US\$10 Billion in 2020, Says ABI Research - Business Wire," 2015.

[2] C. Van Slyke, Information communication technologies : concepts, methodologies, tools and applications. Information Science Reference, 2008. 
[3] Y. Zhuang, L. Hua, L. Qi, J. Yang, P. Cao, Y. Cao, Y. Wu, J. Thompson, and H. Haas, "A Survey of Positioning Systems Using Visible LED Lights," IEEE Communications Surveys and Tutorials, no. February, 2018.

[4] H. Liu, H. Darabi, P. Banerjee, and J. Liu, "Survey of wireless indoor positioning techniques and systems," IEEE Transactions on Systems, Man and Cybernetics Part C: Applications and Reviews, vol. 37, no. 6, pp. 1067-1080, 2007.

[5] R. Mautz, "Indoor Positioning Technologies," Ph.D. dissertation, 2012.

[6] P. Krishnamurthy, "Technologies for Positioning in Indoor Areas," in Indoor Wayfinding and Navigation, 2015, pp. 35-51.

[7] M. Agiwal, A. Roy, and N. Saxena, "Next Generation 5G Wireless Networks: A Comprehensive Survey," IEEE Communications Surveys Tutorials, vol. 18, no. 3, pp. 1617-1655, 2016.

[8] L. Feng, R. Q. Hu, J. Wang, P. Xu, and Y. Qian, "Applying VLC in 5G Networks: Architectures and Key Technologies," IEEE Network, no. December, pp. 77-83, 2016

[9] C. Chen, D. A. Basnayaka, and H. Haas, "Downlink performance of optical attocell networks," Journal of Lightwave Technology, vol. 34, no. 1, pp. 137-156, 2016.

[10] K. Yan, H. Zhou, H. Xiao, and X. Zhang, "Current status of indoor positioning system based on visible light," in 2015 15th International Conference on Control, Automation and Systems (ICCAS). IEEE, oct 2015, pp. 565-569.

[11] Q. Xiao, "Range-free and range-based localization of wireless sensor networks," Ph.D. dissertation, The Hong Kong Polytechnic uUniversity, 2011.

[12] Y. Liu and Z. Yang, Location, localization, and localizability : locationawareness technology for wireless networks. Springer, 2014.

[13] N. A. Mohammed and M. A. Elkarim, "Exploring the effect of diffuse reflection on indoor localization systems based on RSSI-VLC," Optics Express, vol. 23, no. 16, p. 20297, 2015.

[14] W. Gu, M. Aminikashani, P. Deng, and M. Kavehrad, "Impact of Multipath Reflections on the Performance of Indoor Visible Light Positioning Systems," Journal of Lightwave Technology, vol. 34, no. 10, 2016.

[15] X. Sun, J. Duan, Y. Zou, and A. Shi, "Impact of multipath effects on theoretical accuracy of TOA-based indoor VLC positioning system,' Photonics Research, vol. 3, no. 6, p. 296, 2015.

[16] G. Shi, Ge Shi, Yong Li, Li Xi, and Baitao Zang, "A Robust Method for Indoor Localization Based on Visible Light Communication," 2016 2nd IEEE International Conference on Computer and Communications (ICCC), pp. 2154-2158, oct 2016.

[17] N. Krommenacker, O. C. Vasquez, M. D. Alfaro, and I. Soto, "A selfadaptive cell-ID positioning system based on visible light communications in underground mines," in 2016 IEEE International Conference on Automatica (ICA-ACCA). IEEE, oct 2016, pp. 1-7.

[18] J. Gao, F. Yang, and X. Ma, "Indoor positioning system based on visible light communication with gray-coded identification," in 2017 13th International Wireless Communications and Mobile Computing Conference (IWCMC). IEEE, jun 2017, pp. 899-903.

[19] T. Komine and M. Nakagawa, "Fundamental analysis for visible-light communication system using LED lights," IEEE Transactions on Consumer Electronics, vol. 50, no. 1, pp. 100-107, feb 2004.

[20] G. Michael and B. Stephen, "CVX: Matlab software for disciplined convex programming, version 2.0 beta." 2013. [Online]. Available: http://cvxr.com/cvx

[21] M. C. Grant and S. P. Boyd, "Graph Implementations for Nonsmooth Convex Programs," in Recent Advances in Learning and Control. London: Springer London, 2008, pp. 95-110. 